\title{
MASSIVE INTESTINAL PNEUMATOSIS AND PNEUMORETROPERITONEUM FOLLOWING HEMATOPOIETIC STEM CELL TRANSPLANTATION IN A 2-YEAR-OLD CHILD
}

\author{
Giorgia Contini ${ }^{1}$, Arianna Bertocchini ${ }^{1}$, Roberto Carta ${ }^{1}$, Pietro Merli ${ }^{2}$, Alessandro \\ Inserra $^{3}$, Pietro Bagolan ${ }^{4}$, and Francesco Morini ${ }^{4}$ \\ ${ }^{1}$ Ospedale Pediatrico Bambino Gesù \\ ${ }^{2}$ Ospedale Pediatrico Bambino Gesu \\ ${ }^{3}$ Bambino Gesù Pediatric Hospital - IRCCS, Rome \\ ${ }^{4}$ Bambino Gesù Children Hospital
}

July 27, 2020

\begin{abstract}
A 2-year-old boy with severe combined immunodeficiency developed gut graft-versus-host disease (GVHD) after hematopoietic stem cell transplantation (HSCT), associated with massive intestinal pneumatosis (IP), pneumoretroperitoneum (PRP) and pneumomediastinum. His fair clinical conditions allowed conservative management, with progressive normalization of imaging findings. The patient did not require surgery and is alive and in good clinical conditions at follow-up. In children with GVHDrelated IP but good clinical conditions and no signs of peritonitis, IP, is not a mandatory indication for surgery, despite its potentially striking imaging features. Conservative management, with intestinal rest, decompression and antibiotics, often allows regression of the clinical picture.
\end{abstract}

\section{ABSTRACT}

A 2-year-old boy with severe combined immunodeficiency developed gut graft-versus-host disease (GVHD) after hematopoietic stem cell transplantation (HSCT), associated with massive intestinal pneumatosis (IP), pneumoretroperitoneum (PRP) and pneumomediastinum. His fair clinical conditions allowed conservative management, with progressive normalization of imaging findings. The patient did not require surgery and is alive and in good clinical conditions at follow-up.

In children with GVHD-related IP but good clinical conditions and no signs of peritonitis, IP, is not a mandatory indication for surgery, despite its potentially striking imaging features. Conservative management, with intestinal rest, decompression and antibiotics, often allows regression of the clinical picture.

\section{INTRODUCTION}

Graft-versus-host disease (GVHD, is the most common immunological complication of allogeneic hematopoietic stem cell transplantation (HSCT); alloreactive donor T cells recognize recipient's cells as non-self, causing direct and indirect damages to different organs. The most commonly affected organs are the skin, liver and gastrointestinal tract. (1)

GVHD can be classified, based on type of signs/symptoms, time from HSCT and immunological feature, as acute or chronic; moreover, overlap forms rarely occur. Acute GVHD (aGVHD) usually develops 3-5 
weeks after transplantation and can involve the skin, liver, and gastrointestinal tract (GI). Chronic GVHD (cGVHD) can occur de-novo or following acute GVHD, arising several months after HSCT and can involve almost any organ. This complication leads to significant morbidity, reduced quality of life, and decreased overall survival. In children the rate of cGVHD tends to be lower $(20 \%-50 \%)$ than in adults $(60 \%-70 \%)$ (2) and most commonly involves the skin, eyes, oral cavity, gastrointestinal tract, liver, and lungs. Less frequently other organs such as the kidneys or heart may be involved. Manifestations of cGVHD can be of inflammatory type (erythematous rash, diarrhea, mucositis, pulmonary infiltrates) or can have fibrotic features (fasciitis, sicca syndrome, esophageal strictures, sclerotic skin changes and bronchiolitis obliterans).

Rarely, GVHD following hematopoietic stem cell transplantation (HSCT) presents with intestinal pneumatosis (IP). IP is usually an indication for surgery. However, it is important to understand when to pursue conservative management of IP, especially in children with complex medical problems, such as GVHD, also to avoid unnecessary and potentially harmful surgery. (3)

We present the case of a child with GVHD following bone marrow transplantation who developed massive IP with PRP, but no signs of pneumoperitoneum or peritonitis.

\section{CASE DESCRIPTION}

A 2-year-old child with severe combined immunodeficiency underwent HSCT from an unrelated cord blood unit. Three months later he developed aGVHD, with acute pancreatitis and severe abdominal pain. Blood tests showed C-reactive protein (CRP) $11.69 \mathrm{mg} / \mathrm{dl}$ (normal $<0.5 \mathrm{mg} / \mathrm{dl}$ ), procalcitonin $(\mathrm{PCT}) 1.54 \mathrm{ng} / \mathrm{ml}$ (normal $<0.5 \mathrm{ng} / \mathrm{ml}$ ), lipase $1800 \mathrm{IU} / \mathrm{L}$ (normal $<160 \mathrm{IU} / \mathrm{L}$ ). Abdominal x-rays and computed tomography (CT) scan showed marked bowel loops distention, massive intestinal pneumatosis (IP), pneumoretroperitoneum (PRP) and pneumomediastinum (Fig. 1). Despite the radiological findings, his clinical conditions were fair, with abdominal pain and distention but no signs of peritonitis.

In view of his fair clinical conditions and laparotomy-related risks, conservative management was carried out, with intestinal rest, decompression with naso-gastric tube placement, broad-spectrum antibiotics and tight clinical observation (e.g., abdominal x-rays every 24 hours).

After an initial increase of both IP and PRP, imaging findings progressively returned to normal within 12 days (Fig. 2). The patient did not require abdominal surgery and is alive at 6 months of follow-up; however, despite several line of immunosuppressive therapy he still presents recurrent flare of GI GVHD.

\section{DISCUSSION}

We report on a child with IP, PRP and pneumomediastinum associated to GVHD following HSCT.

IP is defined as the presence of gas within the bowel wall. In some cases, gas may be also observed in the intraperitoneal and extraperitoneal spaces, and sometimes is accompanied by a local and systemic inflammatory reaction. It is not a disease per se but a radiologic and pathologic finding that can be associated with a variety of disorders, including chronic obstructive pulmonary disease, gastrointestinal obstruction, bowel ischemia, neonatal necrotizing enterocolitis, immunodeficiency syndromes, bacterial or viral infections; moreover, it can occur after surgical procedures as well as colonoscopies. IP has also been reported in individuals with acquired immunodeficient states and in those who have undergone bone marrow transplantation, as a manifestation of GVHD. (4) In patient with GVHD after HSCT the pathogenesis of this disorder is unclear and probably multi-factorial. Several elements may be associated with the development of IP, including pre-transplantation chemotherapy and radiotherapy, steroid therapy, infectious colitis and septic shock. (5) GI GVHD, leads to atrophic mucositis with ulcer formation, bacterial and fungal superinfection, fibrosis and development of malabsorption syndromes. Damage to the intestinal mucosa, coexisting infection and inflammatory infiltration with concomitant steroid therapy may predispose to IP. Lymphocyte depletion induced by prolonged steroid therapy causes an alteration of lymphoid follicles. This anatomic and functional defects, associated with the inflammatory process, can produce damage to muscolaris mucosae and allow the passage of intraluminal bacteria or gas into the submucosa. (6) 
IP may be associated with a heterogeneous clinical picture. In some cases it is asymptomatic, but in most patients it presents with abdominal pain, nausea, vomiting and diarrhea. (7) Diagnosis of IP usually comes from an abdominal X-ray. In selected patients, the addition of abdominal CT scan may allow the identification of further signs reflecting the severity of the pathology, such as intestinal wall thickening, other gas collection (such as PRP in our patient), pathological bowel wall or soft tissues contrast enhancement, bowel lumen dilatation, fluid in the peritoneal cavity, gas in the portal vein. (8) In children also abdominal ultrasound can detect intestinal penumatosis and other important signs such as bowel distension, bowel wall thickness, portal venous gas, and free abdominal air as an abdominal x-ray. Moreover abdominal ultrasound can shows free and focal fluid collections, the status of peristalsis, and the presence or absence of bowel wall perfusion using Doppler imaging, with the advantage of the absence of ionizing radiation. However, the results of the latter technique are very operator-dependent. $(9 / 10)$

In our patient, IP was associated with PRP and pneumomediastinum, but not pneumoperitoneum (PP) nor clinical manifestations of peritonitis such as worsening of general conditions, abdominal tenderness, abdominal wall discoloration. The presentation suggested a confined condition, that allowed us to undertake conservative management. Generally, in IP without PP, conservative management, including intravenous antibiotics and bowel rest for at least 7 days, should be the first-line treatment. This is especially true in complex patients such as those with GVHD, where surgical stress may be particularly harmful. Enteral feeding may be gradually re-introduced when laboratory and imaging findings suggest regression of the infectious/inflammatory process. Parenteral nutrition is administered until feeding are fully re-established. (11) Conversely, in most cases of IP with associated PP, emergency surgery is mandatory, as the association of $\mathrm{PP}$ is often synonymous with intestinal perforation and peritonitis, which in turn may be the motor for systemic inflammatory reaction syndrome and multiple organ dysfunction, and PP associated with IP without peritonitis has been only rarely reported in the literature. $(11 / 12)$

In conclusion, in children with GVHD, IP is a rare complication that may represent a conundrum for treating physicians and surgeons. Imaging findings alone may depict a situation that seems more severe than it really is. If clinical conditions are stable, treatment should be as conservative as possible. In these complex patients, surgical exploration should be reserved for those with clinical symptoms of peritonitis, signs of intestinal perforation or obstruction.

\section{CONFLICT OF INTEREST STATEMENT}

All the authors declare that they have no conflict of interest.

\section{REFERENCES:}

1. Ji-Hye Lee, Gye-Yeon Lim, Soo Ah Im, Nak-Gyun Chung, Seung-Tae Hahn. Gastrointestinal Complications Following Hematopoietic Stem Cell Transplantation in Children. Korean J Radiol 2008; 9:449-457

2. Kristin Baird, Kenneth Cooke, Kirk R. Schultz. Chronic Graft-Versus- Host Disease (GVHD) in Children. Pediatr Clin N Am 2010; 57:297-322

3. Andrew D. Jones, Richard Maziarz, Jason Gilster, John Domreis, Clifford W. Deveney, Brett C. Sheppard. Surgical complications of bone marrow transplantation. The American Journal of Surgery 2003; 185:481-484

4. P.N. Khalil, S. Huber-Wagner, R. Ladurner, A. Kleespies, M. Siebeck, W. Mutschler, K. Hallfeldt, K.G. Kanz. Natural history, clinical pattern, and surgical considerations of pneumatosis intestinalis. Eur J Med Res 2009; 14: 231-239

5. Deborah L. Day, Norma K.C. Ramsay, Janis Gissel Letourneau. Pneumatosis Intestinalis After Bone Marrow Transplantation. AJR 1988; 151:8-87

6. C Zu lke, S Ulbrich, C Graeb, J Hahn, M Strotzer, E Holler, and K-W Jauch. Acute pneumatosis cystoides intestinalis following allogeneic transplantation the surgeon's dilemma. Bone Marrow Transplantation 2002; 29:795-798

7. Newman JA, Candfield S, Howlett D, McKenzie P, Sahu S. Graft-versus-host disease. BMJ Case 
Reports 2010; 10:1136

8. Katarzyna Laskowska, Małgorzata Burzyńska-Makuch, Anna Krenska, Sylwia Kołtan, Małgorzata Chrupek, Elżbieta Nawrocka, Władysław Lasek, Zbigniew Serafin. Pneumatosis cystoides interstitialis: A complication of graft-versus-host disease. A report of two cases. Pol J Radiol 2012; 77:60-63

9. Takahiro Hosokawa, Yoshitake Yamada, Yutaka Tanami et al. Predictors of prognosis in children with portal venous gas detected by ultrasound. Med Ultrason 2019; 21:30-36

10. Shuai Chen, Yuanjun $\mathrm{Hu}$, Qinghua Liu et al. Application of abdominal sonography in diagnosis of infants with necrotizing enterocolitis. Medicine 2019; 98:28

11. N. Ade-Ajayi, P. Veys, M. Stanton, D.P. Drake, A. Pierro. Conservative management of pneumatosis intestinalis and pneumoperitoneum following bone-marrow transplantation. Pediatr Surg Int 2002; 18:692-695

12. Takanashi M, Hibi S, Todo S, Sawada T, Tsunamoto K, Imashuku S. Pneumatosis cystoides intestinalis with abdominal free air in a 2-year-old girl after allogeneic bone marrow transplantation. Pediatr Hematol Oncol 1998; 15:81-84

\section{LEGENDS:}

Figure 1: a: abdominal x-rays showing IP; b: coronal CT scan with PRP (arrows); c: sagittal CT scan showing pneumomediastinum (arrow head), PRP (arrow) and IP (empty arrows).

Figure 2: consecutive abdominal x-rays (a: day 1; b: day 3; c: day 5; d: day 11) showing progressive reduction of intestinal pneumatosis. Clinical conditions remained fair during all period.

ethics statement

The patient involved in this case was appropriately consented for this publication using the institution's policy for media consent.

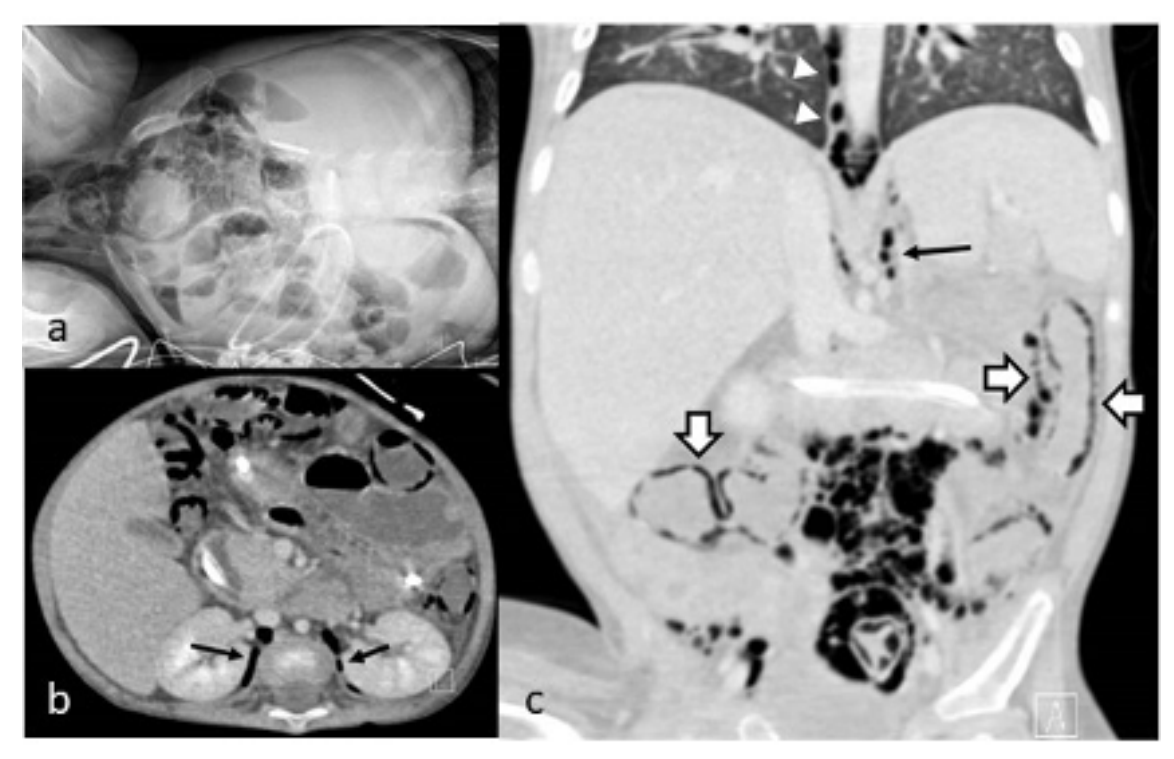




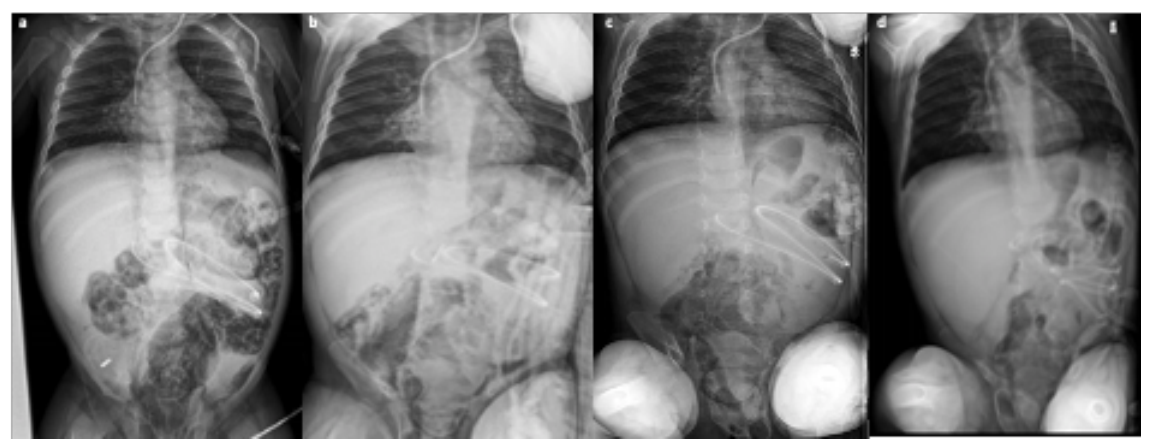

